Al-Azhar Bull. Sci. Vol. 17, No. 2 (Dec.): pp. 93-104, 2006

\title{
SYNTHESIS AND ANTIBACTERIAL ACTIVITY OF SOME NEW \\ PYRAZOLES INCORPORATED WITH IMIDAZOLE, PYRROLE AND OTHER CYCLIC AND HETEROCYCLIC MOIETIES.
}

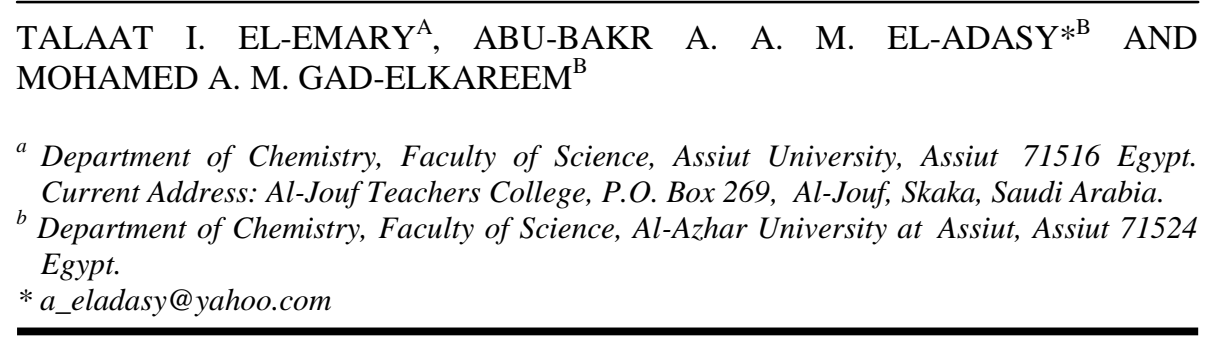

\begin{abstract}
:
Condensation of 1,3-diphenylpyrazole-4-carboxaldehyde (1) with some cyclic and heterocyclic active methylene derivatives afforded the condensation products 2-5. The reaction of 1 with benzoylglycine afforded the key intermediate 4-[(1,3-diphenyl- $1 \mathrm{H}$-pyrazol4-yl)methylene]-2-phenyl-1,3-oxazol-5(4H)-one (6). Also, 1 reacted with malononitrile to give 7. The later reacted with $1 \mathrm{H}$-indene-1,3(2H)-dione to give 9. The oxazolone 6 reacted with some aromatic and heteroaromatic amine derivatives to yield 5-[(1H-pyrazol-4yl)methylene]-3,5-dihydro-4H-imidazol-4-one derivatives 11a,b, 12, 13 and 15. Reacting the oxazolone 6 with acetylacetone, ethyl acetoacetate, 5,5-dimethylcyclohexane-1,3-dione and $1 H$-indane-1,3-(2H)-dione gave the $1 H$-pyrazol-4-ylmethylenepyrrolidine-2,4-dione, $1 H$ pyrazol-4-ylmethylenepyrrol-3-one, $1 H$-pyrazol-4-ylmethyleneindole-3,4-dione and $1 \mathrm{H}$ pyrazol-4-ylmethyleneindeno[1,2- $b$ ]pyrrole-3,4-dione derivatives 18-21 respectively. Antibacterial activity of some of the newly synthesized compounds was studded.
\end{abstract}

Keywords: 1,3-Diphenylpyrazole, oxazolone, pyrrolone, imidazolone, indanedione and antibacterial.

\section{Introduction}

In the recent years a lot of attention on the synthesis and chemistry of pyrazoles has been reported ${ }^{1-6}$ because of their significant and versatile biological and pharmacological activities, such as anti-leishmanial ${ }^{7}$, antimalarial ${ }^{8}$, analgesic and anti-inflammatory ${ }^{9}$, photo-antiproliferative ${ }^{10}$, antibacterial and antifungal ${ }^{2,11-15}$, as potent cannabinoid CB1 receptor antagonists ${ }^{16,17}$. Has potential glucocorticoid receptor ligand for positron emission tomography $(\mathrm{PET})^{18}$. As well, have good properties as anticancer ${ }^{19}$ and antiparasitic ${ }^{20,21}$. Recently, imidazoles were used as Potent anticytokine agents with low activity against hepatic cytochrome P450 
enzymes $^{22}$, antithrombotics ${ }^{23}$, cardioprotective ${ }^{24}$, antibacterial ${ }^{25}$, agents and kinase inhibitors $^{26}$. Moreover, pyrroles were used as antiviral ${ }^{27}$, antiepileptic ${ }^{28}$, anticoccidial $^{29}$, antibacterial ${ }^{30}$ agents and histone deacetylase inhibitors ${ }^{31}$. In continuation to our pervious work directed towards the synthesis and reactions of substituted pyrazoles ${ }^{1,2,6,13-15,20,32}$ and imidazoles ${ }^{33-35}$, we report, here, a new and convenient method for the synthesis and antibacterial activities of some new pyrazole incorporated with imidazole, pyrrole and other cyclic and heterocyclic moieties utilizing 1,3-diphenyl-1 $H$-pyrazole-4-carboxaldehyde.

\section{Results And Discussion}

Condensation of 1,3-diphenylpyrazole-4-carboxaldehyde $(\mathbf{1})^{36}$ with some cyclic active methylene compounds such as cyclohexanone, 5,5-dimethylcyclohexane-1,3dione, $1 H$-indene-1,3(2H)-dione and 3-methyl-2,4-dihydro-3H-pyrazol-5-one yielded the condensation products 2-[(1,3-diphenyl- $1 H$-pyrazol-4yl)methylene]cyclohexanone (2), 2-[(1,3-diphenyl-1H-pyrazol-4-yl)methylene]-5,5dimethylcyclohexane-1,3-dione (3), 2-[(1,3-diphenyl-1 $H$-pyrazol-4-yl)methylene]$1 H$-indane-1,3(2H)-dione (4) and 4-[(1,3-diphenyl-1 $H$-pyrazol-4-yl)methylene]-5methyl-2,4-dihydro-3H-pyrazol-3-one (5) respectively (Scheme 1).

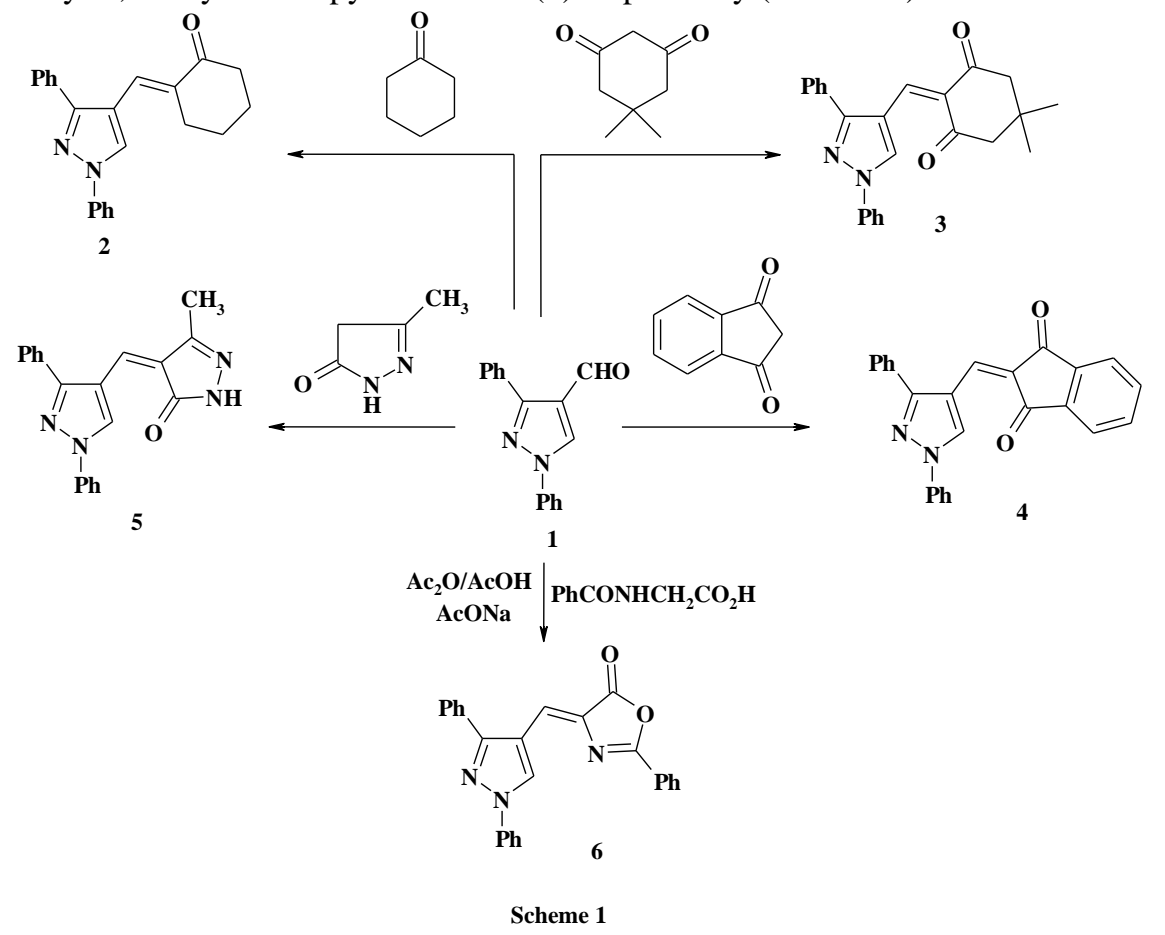


Reaction of 1 with benzoylglycine in acetic acid /acetic anhydride in presence of fused sodium acetate under reflux afforded 4-[(1,3-diphenyl-1 $H$-pyrazol-4yl)methylene]-2-phenyl-1,3-oxazol-5(4H)-one (6). The IR spectrum of 6 indicated the presence of $\mathrm{C}=\mathrm{O}$ at $v=1790 \mathrm{~cm}^{-1}$, and its ${ }^{1} \mathrm{H}$ NMR revealed $\delta=7.02(\mathrm{~s}, 1 \mathrm{H}$, ethylenic-H), 7.42-7.95 (m, 15H, Ar-H) and 9.10 (s, 1H, pyrazole-H) (Scheme 1).

Condensing 1 with malononitrile gave [(1,3-diphenyl-1 $H$-pyrazol-4yl)methylene]malononitrile (7) ${ }^{14}$. Reacting 7 with $1 H$-indane-1,3(2H)-dione in DMF containing equivalent amount of $\mathrm{KOH}$ afforded 4-(1,3-diphenyl-1 $H$-pyrazol-4-yl)2,5-dioxo-2,5-dihydro- $1 H$-indeno[1,2-b]pyridine-3-carbonitrile (9). The structure of compound 9 was confirmed by elemental analysis and spectral data. Its IR spectrum indicated the presence of $\mathrm{NH}$ at $v=3386 \mathrm{~cm}^{-1}, \mathrm{CN}$ at $v=2223 \mathrm{~cm}^{-1}$ and two $\mathrm{C}=\mathrm{O}$ at $v=1722,1679 \mathrm{~cm}^{-1}$, and its ${ }^{1} \mathrm{H}$ NMR revealed 7.37-8.00 (m, 14H, Ar-H), 9.05 (s, $1 \mathrm{H}$, pyrazole-H) and $10.07(\mathrm{~s}, 1 \mathrm{H}, \mathrm{NH})$. The formation of 9 is assumed to proceed via the intermediate 8 , which underwent Dimorth rearrangment ${ }^{37}$ under the applied reaction condition to give $\mathbf{9}$ (Scheme 2).

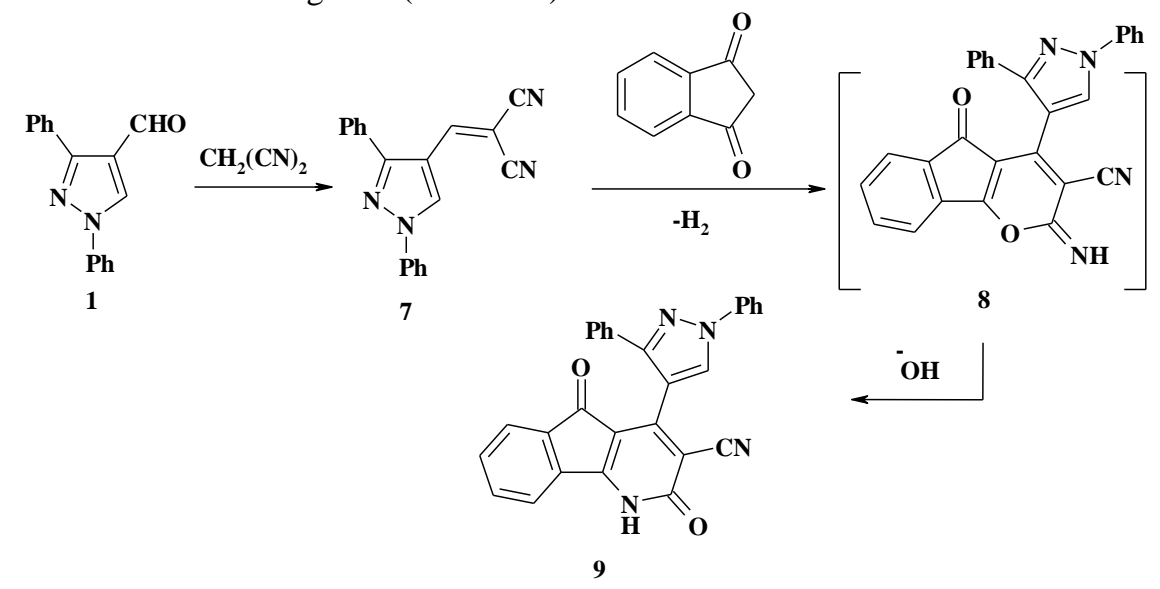

Scheme 2

The oxazolone $\mathbf{6}$ was used as key intermediate for the synthesis of the some imidazole derivatives via its reaction with some aromatic and heteroaromatic amines. Thus, 6 reacted with 2 -aminophenol (10a) in glacial acetic acid solution in presence of fused sodium acetate to produce 5-[(1,3-diphenyl-1H-pyrazol-4yl)methylene]-3-(2-hydroxyphenyl)-2-phenyl-3,5-dihydro-4H-imidazol-4-one (11a). The IR spectrum of 11a indicated the presence of $\mathrm{OH}$ at $v=3350 \mathrm{~cm}^{-1}$ and $\mathrm{C}=\mathrm{O}$ at $v$ $=1653 \mathrm{~cm}^{-1}$, while its ${ }^{1} \mathrm{H}$ NMR revealed $\delta=7.10(\mathrm{~s}, 1 \mathrm{H}$, ethylenic- $\mathrm{H}), 7.25-7.97(\mathrm{~m}$, 19H, Ar-H), 9.40 (s, 1H, pyrazole-H) and 10.01 (s, 1H, OH) (Scheme 3). 


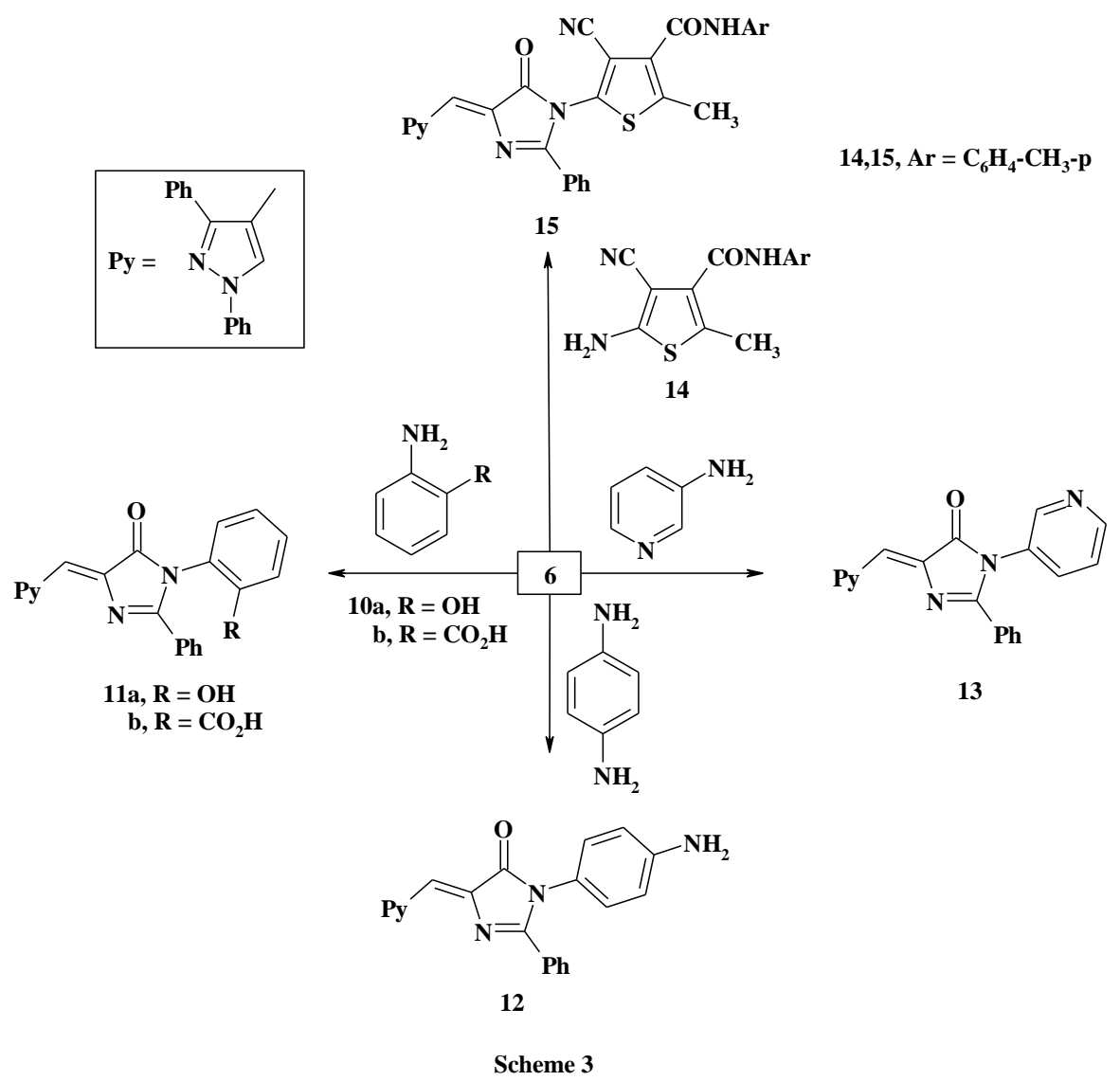

Similarly 6 reacted with 2 -aminobenzoic acid $(\mathbf{1 0 b})$ and p-phenylenediamine to give 2-\{4-[(1,3-diphenyl-1 $H$-pyrazol-4-yl)methyl-ene]-5-oxo-2-phenyl-4,5-dihydro$1 H$-imidazol-1-yl benzoic acid (11b) and 3-(4-aminophenyl)-5-[(1,3-diphenyl- $1 H$ pyrazol-4-yl)methylene]-2-phenyl-3,5-dihydro-4H-imida-zol-4-one (12) respectively (Scheme 3).

As well, 6 reacted with some heteroaromatic amines as 3-aminopyridine and 5amino-4-cyano-2-methyl- $N$-(4-methyl-phenyl)thiophene-3-carboxamide (14) to yield 5-[(1,3-diphenyl-1H-pyrazol-4-yl)methylene]-2-phenyl-3-pyridin-3-yl-3,5dihydro-4H-imidazol-4-one (13) and 4-cyano-5-\{4-[(1,3-diphenyl-1H-pyrazol-4-yl)methylene]-5-oxo-2-phenyl-4,5-dihydro- $1 H$-imidazol-1-yl $\}-N$-(4-methyl-phenyl)-2methylthiophene-3-carboxamide (15) respectively (Scheme 3).

The strategy has also extended to synthesis new pyrrolones moieties attached to 1,3-diphenylpyrazole nucleuses at position -4 . Thus, on reacting the oxazolone 6 
with acetylacetone in dioxan in presence of sodium metal ${ }^{38}$, it afforded 4-acetyl-2[(1,3-diphenyl-1 $H$-pyrazol-4-yl)methylene]-5-methyl-1,2-dihydro-3 $H$-pyrrol-3-one (18). The IR spectrum of 18 indicated the presence of NH at $v=3279 \mathrm{~cm}^{-1}$ and two $\mathrm{C}=\mathrm{O}$ at $v=1702,1643 \mathrm{~cm}^{-1}$, and its ${ }^{1} \mathrm{H}$ NMR revealed $\delta=2.05\left(\mathrm{~s}, 3 \mathrm{H}, \mathrm{COCH}_{3}\right)$, $3.22\left(\mathrm{~s}, 3 \mathrm{H}, \mathrm{CH}_{3}\right), 7.32$ (s, $1 \mathrm{H}$, ethylenic-H), 7.38-8.01 (m, 10H, aromatic-H), 8.72 $(\mathrm{s}, 1 \mathrm{H}$, pyrazole-H) and $10.00(\mathrm{~s}, 1 \mathrm{H}, \mathrm{NH})$ (Scheme 4$)$. The formation of $\mathbf{1 8}$ is assumed to take place via the corresponding nonisolable intermediate 16, which readily cyclizes under the reaction conditions to give $\mathbf{1 7}$. The latter compound undergoes debenzoylation ${ }^{38}$ to give the final product 18 (Scheme 4).

Finally, 6 reacted with: ethyl acetoacetate, 5,5-dimethylcyclohexane-1,3-dione and $1 H$ indane-1,3(2H)-dione to achieved 3-acetyl-5-[(1,3-diphenyl-1H-pyrazol-4yl)methylene]pyrrolidine-2,4-dione (19), 6,6-dimethyl-2-[(1,3-diphenyl-1 $H$-pyrazol4-yl)methylene]-6,7-dihydro- $1 H$-indole-3,4(2H, 5H)-dione (20) and 2-[(1,3diphenyl-1 $H$-pyrazol-4-yl)methylene]-1,2-dihydro-indeno[1,2- $b]$ pyrrole-3,4-dione (21) respectively. The structure of compounds 18-21 was established based on elemental analyses, spectral data and literature ${ }^{38}$ reports (Scheme 4).

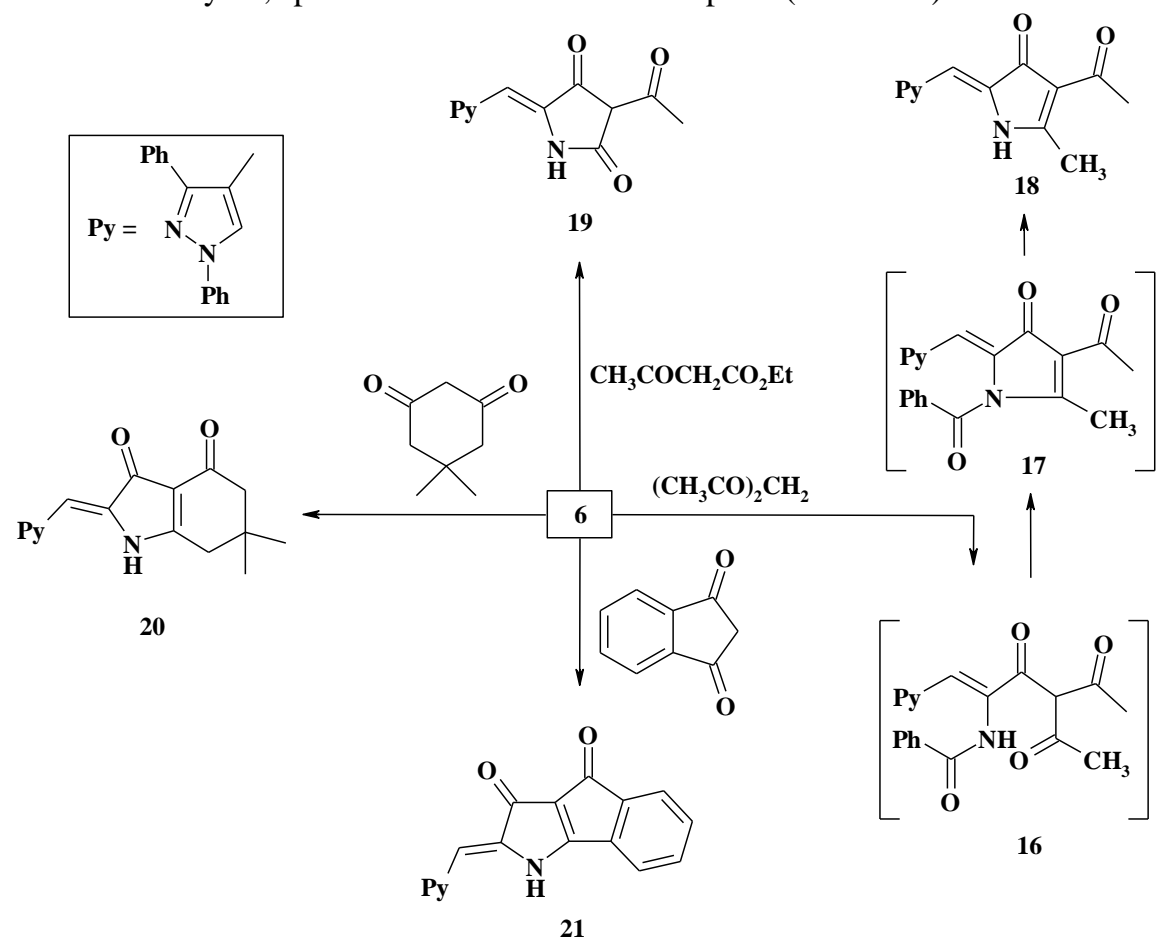

Scheme 4 


\section{Antibacterial Activity}

Ten of the synthesized compounds were screened in vitro for their antibacterial activities against three strains of bacteria (Escherichia Coli, Pseudomonas areuginosa and Klebsiella pneumonia) using the filter paper disc technique ${ }^{39}$. The results are given in Table III. The screened compounds were dissolved in DMF to get a solution of $1 \%$ concentration. Filtered paper discs (Whatman No. 1 filter paper, $5 \mathrm{~mm}$ diameter) were saturated with this solution. The discs were placed on the surface solidified nutrient agar dishes seeded by the tested bacteria. Inhibition zones were measured at the end of an incubation period of $48 \mathrm{~h}$. (at $37^{\circ} \mathrm{C}$ ). Tioconazole (Tyrosyd) was used as reference substance. Most compounds showed from moderate to strong activities against klebsiella Pneumonia. Compounds 4, 18, 19 and 21 showed strong activities against Pseudomonas arenginosa. Compounds 6, 11a, 15, 19 and 21 showed from weak to moderate activities against Escherichia coli. The rest of the compounds exhibited nil activities against all strains of bacteria used.

\section{Exepremental}

Melting points were measured with a Gallenkamp apparatus and are uncorrected. IR spectra in $\mathrm{KBr}$ discs were recorded on BRUKER Vector 22 FT-IR spectrophotometer. ${ }^{1} \mathrm{H}$ NMR spectra were determined in DMSO- $d_{6}$ at $300 \mathrm{MHz}$ on Varian Mercury VX spectrometer using TMS as an internal standard. Chemical shifts are expressed as $\delta$ ppm. Elemental analyses were carried out at the Microanalytical Center of Cairo University. Mass spectra were recorded on a Finnigan Mat SSQ-7000 apparatus at $70 \mathrm{eV}$. Compounds $\mathbf{1}^{36}$ and $7^{14}$ were prepared according to the literature procedures.

\section{Condensation of 1 with cyclic active methylene compounds:}

\section{General procedure}

A mixture of $1(0.01 \mathrm{~mol})$ and cyclic active methylene compounds namely: cyclohexanone, 5,5-dimethyl-cyclohexane-1,3-dione, $1 H$-indene-1,3(2H)-dione and 5-methyl-2,4-di-hydro-3H-pyrazol-3-one $(0.01 \mathrm{~mol})$ in ethanol $(30 \mathrm{~mL})$ and catalytic amount of triethyl amine $(0.5 \mathrm{~mL})$ was heated under reflux for $3 \mathrm{~h}$. The solid precipitate that formed was collected, dried and recrystallized from the proper solvents to give 2-5 respectively (Tables I and II).

Reaction of 1 with benzoylglycine: Formation of 4-((1,3-diphenyl-1H-pyrazol-4yl)methylene)-2-phenyl-1,3-oxazol-5(4H )-one (6).

A mixture of $1(0.01 \mathrm{~mol})$, benzoylglycine $(0.01 \mathrm{~mol})$ and fused sodium acetate $(0.03 \mathrm{~mol})$ in acetic acid $(10 \mathrm{~mL})$ and acetic anhydride $(10 \mathrm{~mL})$ was heated on a 
steam bath for $3 \mathrm{~h}$. The reaction mixture was then poured into a cold mixture of ethanol-water $(1: 1)(50 \mathrm{ml})$ and stirred for $15 \mathrm{~min}$. The solid precipitate that formed was collected, washed with cold ethanol, dried and recrystallized from acetic acid to give 6 (Tables I and II).

Reaction of 7 with $1 \mathrm{H}$-indane-1,3(2H)-dione. Formation of 4-(1,3-diphenyl-1Hpyrazol-4-yl)-2,5-dioxo-2,5-dihydro-1H-indeno[1,2-b]pyridine-3-carbonitrile (9).

A mixture of compound $7(0.01 \mathrm{~mol})$ and $1 H$-indane-1,3(2H)-dione $(0.01 \mathrm{~mol})$ in DMF $(30 \mathrm{~mL})$ containing $0.01 \mathrm{~mol} \mathrm{KOH}$ was heated under reflux for $5 \mathrm{~h}$. The reaction mixture was cooled, poured onto ice- water and neutralized with dil. $\mathrm{HCl}$. The solid product that formed was filtered off, dried and crystallized from dioxan to give 9 (Tables I and II).

Reaction of 6 with aromatic and hetero-aromatic amines: Formation of 11a, 11b, 12,13 and 15.

\section{General procedure}

A mixture of 6 (0.01 mol) and aromatic or hetero-aromatic amines namely: 2aminophenol (10a), 2-aminobenzoic acid (10b), 3-aminopyridine, pphenylenediamine and 5-amino-4-cyano-2-methyl- $N$-(4-methylphenyl) thio-phene3 -carboxamide (14) $(0.01 \mathrm{~mol}))$ and fused sodium acetate $(0.03 \mathrm{~mol})$ in glacial acetic acid $(30 \mathrm{~mL})$ was heated under reflux for $1 \mathrm{~h}$., then cooled. The reaction mixture was poured onto cold water. The solid products were filtered off, washed with water, dried and recrystallized from the proper solvents to give 11a, 11b, 12,13 and $\mathbf{1 5}$ respectively (Tables I and II).

Reaction of 6 with: acetylacetone, ethyl acetoacetate, 5,5-dimethyl-cyclohexane1,3-dione and $1 \mathrm{H}$-indene-1,3(2H)-dione: Formation of compounds 18- 21.

\section{General procedure}

A finely divided sodium metal $(0.01 \mathrm{~mol})$ was added to a solution of the appropriate active methylene compounds $(0.01 \mathrm{~mol})$ in dioxan $(30 \mathrm{~mL})$ and the mixture was stirred at room temperature for $24 \mathrm{~h}$., after that compound $6(0.01 \mathrm{~mol})$ was added. The reaction mixtures were heated under reflux for $8 \mathrm{~h}$. The reaction mixtures were then poured onto ice-cold water and acidified with dilute $\mathrm{HCl}$. The solid products that formed were collected, washed with water, dried and crystallized from the proper solvent to give 18-21 (Table I and II). 


\section{Acknowledgment}

The authors are grateful to Botany and Microbiology Department, Faculty of Science, Al-Azhar University, Assiut, Egypt for doing the Antibacterial activities tests.

Table I: Characterization data of the newly synthesized compounds.

\begin{tabular}{|c|c|c|c|c|c|c|}
\hline \multirow{2}{*}{$\begin{array}{l}\text { Compd. } \\
\text { No. }\end{array}$} & \multirow{2}{*}{$\frac{\text { M.P. }\left({ }^{\circ} \mathrm{C}\right)}{\text { Yield }(\%)}$} & \multirow{2}{*}{$\frac{\text { Solvent Cryst. }}{(\text { Colour })}$} & \multirow{2}{*}{$\frac{\text { Mol. Formula }}{\text { (Mol. Wt) }}$} & \multicolumn{3}{|c|}{$\begin{array}{l}\text { Elemental analysis } \\
\text { calculated / found \% }\end{array}$} \\
\hline & & & & $\mathrm{C}$ & $\mathrm{H}$ & $\mathrm{N}$ \\
\hline \multirow{2}{*}{2} & 240 & Dioxan & $\mathrm{C}_{22} \mathrm{H}_{20} \mathrm{~N}_{2} \mathrm{O}$ & 80.46 & 6.14 & 8.53 \\
\hline & (74) & (Yellow) & (328) & 80.58 & 6.32 & 8.51 \\
\hline \multirow{2}{*}{3} & 140 & Ethanol & $\mathrm{C}_{24} \mathrm{H}_{22} \mathrm{~N}_{2} \mathrm{O}_{2}$ & 77.82 & 5.99 & 7.56 \\
\hline & (68) & (Yellow) & $(370.4)$ & 77.50 & 6.23 & 7.45 \\
\hline \multirow{2}{*}{4} & 295 & Dioxan & $\mathrm{C}_{25} \mathrm{H}_{16} \mathrm{~N}_{2} \mathrm{O}_{2}$ & 79.77 & 4.28 & 7.44 \\
\hline & (73) & (Yellow) & (376.4) & 79.52 & 4.48 & 7.43 \\
\hline \multirow{2}{*}{5} & 238 & Ethanol & $\mathrm{C}_{20} \mathrm{H}_{16} \mathrm{~N}_{4} \mathrm{O}$ & 73.14 & 4.91 & 17.06 \\
\hline & (61) & (Yellow) & (328) & 73.32 & 4.83 & 17.22 \\
\hline \multirow{2}{*}{6} & 175 & Acetic acid & $\mathrm{C}_{25} \mathrm{H}_{17} \mathrm{~N}_{3} \mathrm{O}_{2}$ & 76.71 & 4.38 & 10.74 \\
\hline & (73) & (Yellow) & (391.4) & 76.85 & 4.33 & 10.68 \\
\hline \multirow{2}{*}{9} & 228 & Dioxan & $\mathrm{C}_{28} \mathrm{H}_{16} \mathrm{~N}_{4} \mathrm{O}_{2}$ & 76.34 & 3.66 & 12.72 \\
\hline & (61) & (Yellow) & (440.5) & 76.58 & 3.68 & 12.82 \\
\hline \multirow{2}{*}{$11 \mathrm{a}$} & 150 & Ethanol & $\mathrm{C}_{31} \mathrm{H}_{22} \mathrm{~N}_{4} \mathrm{O}_{2}$ & 77.16 & 4.60 & 11.61 \\
\hline & (56) & (Yellow) & $(482.5)$ & 77.34 & 4.53 & 11.94 \\
\hline \multirow{2}{*}{$11 b$} & 145 & Dioxan & $\mathrm{C}_{32} \mathrm{H}_{22} \mathrm{~N}_{4} \mathrm{O}_{3}$ & 75.28 & 4.34 & 10.98 \\
\hline & (51) & (Yellow) & (510.5) & 75.36 & 4.11 & 11.23 \\
\hline \multirow{2}{*}{12} & 310 & Dioxan & $\mathrm{C}_{31} \mathrm{H}_{23} \mathrm{~N}_{5} \mathrm{O}$ & 77.31 & 4.81 & 14.55 \\
\hline & (71) & (Yellow) & (481.6) & 77.54 & 4.95 & 14.86 \\
\hline \multirow{2}{*}{13} & 250 & Ethanol & $\mathrm{C}_{30} \mathrm{H}_{21} \mathrm{~N}_{5} \mathrm{O}$ & 77.07 & 4.53 & 14.98 \\
\hline & (66) & (Yellow) & $(467.5)$ & 77.23 & 4.62 & 14.77 \\
\hline \multirow{2}{*}{15} & 210 & Ethanol & $\mathrm{C}_{39} \mathrm{H}_{28} \mathrm{~N}_{6} \mathrm{O}_{2} \mathrm{~S}$ & 72.65 & 4.38 & 13.04 \\
\hline & (64) & (Yellow) & $(644.7)$ & 72.77 & 4.56 & 13.11 \\
\hline \multirow{2}{*}{18} & 220 & Ethanol & $\mathrm{C}_{23} \mathrm{H}_{19} \mathrm{~N}_{3} \mathrm{O}_{2}$ & 74.78 & 5.18 & 11.37 \\
\hline & (46) & (Yellow) & $(369.4)$ & 74.82 & 5.34 & 11.68 \\
\hline \multirow{2}{*}{19} & 228 & Ethanol & $\mathrm{C}_{22} \mathrm{H}_{17} \mathrm{~N}_{3} \mathrm{O}_{3}$ & 71.14 & 4.61 & 11.32 \\
\hline & (68) & (Yellow) & (371.4) & 71.34 & 4.68 & 11.65 \\
\hline \multirow{2}{*}{20} & 132 & Ethanol & $\mathrm{C}_{26} \mathrm{H}_{23} \mathrm{~N}_{3} \mathrm{O}_{2}$ & 76.25 & 5.66 & 10.26 \\
\hline & (58) & (Yellow) & (409.5) & 76.43 & 5.98 & 10.34 \\
\hline \multirow{2}{*}{21} & 230 & Dioxan & $\mathrm{C}_{27} \mathrm{H}_{17} \mathrm{~N}_{3} \mathrm{O}_{2}$ & 78.06 & 4.13 & 10.12 \\
\hline & (73) & (Yellow) & (415.4) & 78.12 & 4.21 & 10.32 \\
\hline
\end{tabular}


Table II: Spectral data of the newly synthesized compounds.

\begin{tabular}{|c|c|}
\hline $\begin{array}{l}\text { Compd. } \\
\text { No. }\end{array}$ & $\begin{array}{c}\text { Spectral data: IR (KBr) } v_{\max }\left(\mathrm{cm}^{-1}\right),{ }^{1} \mathrm{H} \text { NMR } \delta \mathrm{ppm} \text { and } \\
\text { Ms: } \mathrm{m} / \mathrm{z}(\%)\end{array}$ \\
\hline 2 & $\begin{array}{l}\text { IR v: } 1657(\mathrm{CO}) .{ }^{1} \mathrm{H}-\mathrm{NMR}\left(\mathrm{DMSO}-d_{6}\right) \delta: 1.33\left(\mathrm{~m}, 2 \mathrm{H}, \mathrm{CH}_{2} \text {-cyclohexane }\right), 1.40(\mathrm{~m} \text {, } \\
2 \mathrm{H}, \mathrm{CH}_{2} \text {-cyclohexane), } 1.96\left(\mathrm{t}, 2 \mathrm{H}, \mathrm{CH}_{2}-\mathrm{cyclohexane}\right), 2.94\left(\mathrm{t}, 2 \mathrm{H}, \mathrm{CH}_{2}-\mathrm{cyclohexane}\right) \text {, } \\
7.15(\mathrm{~s}, 1 \mathrm{H} \text {, ethylene-H), } 7.43-7.68(\mathrm{~m}, 10 \mathrm{H}, \mathrm{Ar}-\mathrm{H}) \text { and } 9.34(\mathrm{~s}, 1 \mathrm{H}, \text { pyrazole-H). }\end{array}$ \\
\hline 3 & $\begin{array}{l}\text { IR v: } 1673,1655(2 \mathrm{CO}) .{ }^{1} \mathrm{H}-\mathrm{NMR}\left(\mathrm{DMSO}-\mathrm{d}_{6}\right) \delta: 1.49\left(\mathrm{~s}, 6 \mathrm{H}, 2 \mathrm{CH}_{3}\right), 2.21(\mathrm{~s}, 4 \mathrm{H} \text {, } \\
2 \mathrm{CH}_{2} \text {-cyclohexane), 7.45-7.70 (m, 10H, Ar-H), } 7.30(\mathrm{~s}, 1 \mathrm{H} \text {, ethylene-H) and } 9.15(\mathrm{~s} \text {, } \\
1 \mathrm{H}, \text { pyrazole-H). }\end{array}$ \\
\hline 4 & $\begin{array}{l}\text { IR v: } 1708,1677(2 \mathrm{CO}) .{ }^{1} \mathrm{H}-\mathrm{NMR}\left(\mathrm{DMSO}_{-}\right) \delta: 7.43-7.76(\mathrm{~m}, 14 \mathrm{H}, \mathrm{Ar}-\mathrm{H}), 7.26(\mathrm{~s}, \\
1 \mathrm{H} \text {, ethylene-H) and } 9.01\left(\mathrm{~s}, 1 \mathrm{H}, \text { pyrazole-H). Ms: } \mathrm{m} / \mathrm{z}(\%), 376\left(\mathrm{M}^{+}, 35.6\right), 375(100) \text {, }\right. \\
331(18), 276(14.6), 215(13.6), 140(9.1), 105(10), 77(59.5) \text { and } 51(12.5) .\end{array}$ \\
\hline 5 & $\begin{array}{l}\text { IR v: } 3118(\mathrm{NH}) \text { and } 1670(\mathrm{CO}) .{ }^{1} \mathrm{H}-\mathrm{NMR}\left(\mathrm{DMSO}-d_{6}\right) \delta: 2.35\left(\mathrm{~s}, 3 \mathrm{H}, \mathrm{CH}_{3^{-}}\right. \\
\text {pyrazolone }), 7.00(\mathrm{~s}, 1 \mathrm{H}, \mathrm{NH}), 7.42-7.68(\mathrm{~m}, 10 \mathrm{H}, \mathrm{Ar}-\mathrm{H}), 7.10(\mathrm{~s}, 1 \mathrm{H}, \text { ethylene-H) and } \\
9.11(\mathrm{~s}, 1 \mathrm{H}, \mathrm{pyrazole}-\mathrm{H}) . \mathrm{Ms}: \mathrm{m} / \mathrm{z}(\%), 328\left(\mathrm{M}^{+}, 4\right), 273(14.7), 247(31.6), 191(4.8), \\
105(33.4), 77(100) \text { and } 51(30.7) \text {. }\end{array}$ \\
\hline 6 & $\begin{array}{l}\text { IR v: } 1790(\mathrm{CO}) .{ }^{1} \mathrm{H}-\mathrm{NMR}\left(\mathrm{DMSO}-d_{6}\right) \delta: 7.02(\mathrm{~s}, 1 \mathrm{H} \text {, ethylene-H), } 7.42-7.95(\mathrm{~m}, 15 \mathrm{H} \text {, } \\
\text { Ar-H) and } 9.10(\mathrm{~s}, 1 \mathrm{H} \text {, pyrazole-H). }\end{array}$ \\
\hline 9 & $\begin{array}{l}\text { IR v: } 3386(\mathrm{NH}), 2223(\mathrm{CN}), 1722(\mathrm{CO}) \text { and } 1679(\mathrm{CO}) .{ }^{1} \mathrm{H}-\mathrm{NMR}\left(\mathrm{DMSO}-d_{6}\right) \delta: 7.37- \\
8.00(\mathrm{~m}, 14 \mathrm{H}, \mathrm{Ar}-\mathrm{H}), 9.05(\mathrm{~s}, 1 \mathrm{H}, \text { pyrazole-H) and } 10.07(\mathrm{~s}, \mathrm{H}, \mathrm{NH}) .\end{array}$ \\
\hline $11 a$ & $\begin{array}{l}\text { IR v: } 3350(\mathrm{OH}), 1653(\mathrm{CO}) .{ }^{1} \mathrm{H}-\mathrm{NMR}\left(\mathrm{DMSO}-d_{6}\right) \delta: 7.10(\mathrm{~s}, 1 \mathrm{H}, \text { ethylene-H), } 7.25- \\
7.97(\mathrm{~m}, 19 \mathrm{H}, \mathrm{Ar}-\mathrm{H}), 9.40(\mathrm{~s}, 1 \mathrm{H}, \text { pyrazole-H) and } 10.01(\mathrm{~s}, 1 \mathrm{H}, \mathrm{OH}) .\end{array}$ \\
\hline 11b & $\begin{array}{l}\text { IR v: } 3455-3250\left(\text { br. OH), 1670, } 1650(2 \mathrm{CO}) .{ }^{1} \mathrm{H}-\mathrm{NMR}\left(\mathrm{DMSO}-d_{6}\right) \delta: 7.42-8.08(\mathrm{~m} \text {, }\right. \\
19 \mathrm{H}, \mathrm{Ar}-\mathrm{H}), 7.10(\mathrm{~s}, 1 \mathrm{H} \text {, ethylene-H), } 9.11(\mathrm{~s}, 1 \mathrm{H}, \text { pyrazole-H) and } 10.10(\mathrm{~s}, 1 \mathrm{H}, \mathrm{OH})\end{array}$ \\
\hline 12 & $\begin{array}{l}\text { IR } v: 3381,3290\left(\mathrm{NH}_{2}\right) \text { and } 1642(\mathrm{CO}) .{ }^{1} \mathrm{H}-\mathrm{NMR}\left(\mathrm{DMSO}-d_{6}\right) \delta: 7.35-8.60(\mathrm{~m}, 19 \mathrm{H} \text {, } \\
\text { Ar-H), } 7.22\left(\mathrm{~s}, 1 \mathrm{H}, \text { ethylene-H), } 9.25\left(\mathrm{~s}, 1 \mathrm{H}, \text { pyrazole-H) and } 9.99\left(\mathrm{~s}, 2 \mathrm{H}, \mathrm{NH}_{2}\right) . \mathrm{Ms} \text { : }\right.\right. \\
481\left(\mathrm{M}^{+}, 1 \%\right), 391(10.1), 258(21.2), 155(21.2), 105(86.9), 77(100) \text { and } 51(28.8) \text {. }\end{array}$ \\
\hline 13 & $\begin{array}{l}\text { IR v: } 1655(\mathrm{CO}) .{ }^{1} \mathrm{H}-\mathrm{NMR}\left(\mathrm{DMSO}-d_{6}\right) \delta: 7.26-8.53(\mathrm{~m}, 19 \mathrm{H}, \mathrm{Ar}-\mathrm{H}), 7.11(\mathrm{~s}, 1 \mathrm{H} \text {, } \\
\text { ethylene-H) and } 9.33\left(\mathrm{~s}, 1 \mathrm{H}, \text { pyrazole-H). Ms: } \mathrm{m} / \mathrm{z}(\%), 467\left(\mathrm{M}^{+}, 36.2\right), 409(17.6) \text {, }\right. \\
347(45), 305(57.8), 259(44.6), 181(35), 105(100), 77(74.9) \text { and } 51(14.6) .\end{array}$ \\
\hline 15 & $\begin{array}{l}\text { IR: } 3272(\mathrm{NH}), 2215(\mathrm{CN}) \text { and } 1650,1637(2 \mathrm{CO}) .{ }^{1} \mathrm{H}-\mathrm{NMR}\left(\mathrm{DMSO}-d_{6}\right) \delta: 2.24(\mathrm{~s}, 3 \mathrm{H} \text {, } \\
\left.\mathrm{CH}_{3}\right), 2.44\left(\mathrm{~s}, 3 \mathrm{H}, \mathrm{CH}_{3}\right), 7.13-7.98(\mathrm{~m}, 19 \mathrm{H}, \mathrm{Ar}-\mathrm{H}), 7.11(\mathrm{~s}, 1 \mathrm{H} \text {, ethylene-H), } 8.70(\mathrm{~s}, \\
1 \mathrm{H}, \text { pyrazole-H) and } 9.91(\mathrm{~s}, 1 \mathrm{H}, \mathrm{NH}) .\end{array}$ \\
\hline 18 & $\begin{array}{l}\text { IR v: } 3279(\mathrm{NH}), 1702(\mathrm{CO}) \text { and } 1643(\mathrm{CO}) .{ }^{1} \mathrm{H}-\mathrm{NMR}\left(\mathrm{DMSO}-d_{6}\right) \delta: 2.05(\mathrm{~s}, 3 \mathrm{H} \text {, } \\
\left.\mathrm{COCH}_{3}\right), 3.22\left(\mathrm{~s}, 3 \mathrm{H}, \mathrm{CH}_{3}-\text { pyrrolone }\right), 7.32(\mathrm{~s}, 1 \mathrm{H} \text {, ethylene-H), 7.38-8.01 (m, 10H, } \\
\text { Ar-H), } 8.72(\mathrm{~s}, 1 \mathrm{H}, \text { pyrazole-H), and } 10.00(\mathrm{~s}, 1 \mathrm{H}, \mathrm{NH}) .\end{array}$ \\
\hline 19 & $\begin{array}{l}\text { IR v: } 3281(\mathrm{NH}), 1697,1641 \text { and } 1630(3 \mathrm{CO}) .{ }^{1} \mathrm{H}-\mathrm{NMR}\left(\mathrm{DMSO}-d_{6}\right) \delta: 2.05(\mathrm{~s}, 3 \mathrm{H} \text {, } \\
\left.\mathrm{COCH}_{3}\right), 4.01(\mathrm{~s}, 1 \mathrm{H}, \mathrm{CH}-\mathrm{methine}), 6.39(\mathrm{~s}, 1 \mathrm{H} \text {, ethylene-H), 7.15-7.98 (m, 10H, Ar- } \\
\mathrm{H}), 9.34\left(\mathrm{~s}, 1 \mathrm{H}, \text { pyrazole-H) and } 10.44(\mathrm{~s}, 1 \mathrm{H}, \mathrm{NH}) . \mathrm{Ms}: \mathrm{m} / \mathrm{z}(\%), 371\left(\mathrm{M}^{+}, 100\right), 329\right. \\
(47.3), 259(99.8), 155(42.2), 104(51.1), 77(85.5) \text { and } 51(10.4)\end{array}$ \\
\hline 20 & IR v: $3250(\mathrm{NH}), 1670$ and $1655(2 \mathrm{CO})$ \\
\hline 21 & $\begin{array}{l}\text { IR v: } 3278(\mathrm{NH}), 1715 \text { and } 1680(2 \mathrm{CO}) .{ }^{1} \mathrm{H}-\mathrm{NMR}\left(\mathrm{DMSO}-d_{6}\right) \delta: 7.14(\mathrm{~s}, 1 \mathrm{H} \text {, ethylene- } \\
\mathrm{H}), 7.42-7.99(\mathrm{~m}, 14 \mathrm{H}, \mathrm{Ar}-\mathrm{H}), 9.04(\mathrm{~s}, 1 \mathrm{H} \text {, pyrazole-H) and } 9.73(\mathrm{~s}, 1 \mathrm{H}, \mathrm{NH}) .\end{array}$ \\
\hline
\end{tabular}


Table III: The Antibacterial activity of some newly synthesized compounds. ( Diameter of inhibition zone in $\mathbf{m m}$ )

\begin{tabular}{|c|c|c|c|}
\hline Comp. No. & Escherichia Coli & $\begin{array}{c}\text { Pseudomonas } \\
\text { areuginosa }\end{array}$ & $\begin{array}{c}\text { Klebsiella } \\
\text { Pneumonia }\end{array}$ \\
\hline $\mathbf{4}$ & - & 25 & 20 \\
\hline $\mathbf{6}$ & 9 & - & 10 \\
\hline $\mathbf{1 1 a}$ & 9 & - & - \\
\hline $\mathbf{1 1 b}$ & - & - & 10 \\
\hline $\mathbf{1 2}$ & - & - & 20 \\
\hline $\mathbf{1 3}$ & - & - & - \\
\hline $\mathbf{1 5}$ & 11 & - & 20 \\
\hline $\mathbf{1 8}$ & - & 25 & 10 \\
\hline $\mathbf{1 9}$ & 11 & 20 & 20 \\
\hline $\mathbf{2 1}$ & 9 & 17 & 20 \\
\hline
\end{tabular}

No inhibition (-).

\section{References}

1 M. A. M. GAD-ELKAREEM AND A. O. ABDELHAMID, Afinidad, 61(513), 427 (2004).

2 T. I. EL-EMARY, A. KHALIL, G. A. M. EL-HAG ALI AND A. A. A. M. ELADASY, Phosphorus, Sulfur and Silicon and related elements, 180, 19, (2005).

3 M. E. JUNG, S. J. MIN, K. N. HOUK AND D. ESS, J. Org. Chem. J. Org. Chem. 69(26), 9085, (2004).

4 A. ARMSTRONG, L. H. JONES, J. D. KNIGHT AND R. D. KELSEY, Org. Lett. 17; 7(4), 713, (2005).

5 T. NORRIS, R. COLON-CRUZ, AND D. H. RIPIN, Org. Biomol. Chem. 3(10), 1844, (2005).

6 A. M. HUSSEIN AND T. I. EL-EMARY, J. Chem. Res. (S) 1, 20-21, (1998); M., 0231-0241.

7 A. M.R. BERNARDINO, A. O. GOMES, K. S. CHARRET, A. C.C. FREITAS, G. M.C. MACHADO, M. M. CANTO-CAVALHEIRO, L. L. LEON AND V. F. AMARAL, Eur. J. Med. Chem. 41, 80 (2006).

8 W. CUNICO, C. A. CECHINEL, H. G. BONACORSO, M. A. P. MARTINS, N. ZANATTA, M. V. N. DE SOUZA, I. O. FREITAS, R. P. P. SOARES AND A. U. KRETTLI, Bioorg. Med. Chem. Lett. 16 (3), 649 (2006). 
M. SÜKÜROGLU, B. C. ERGÜN, S. ÜNLÜ, M. F. SAHIN, E. KÜPELI, E. YESILADA, AND E. BANOGLU, Arch. Pharm. Res. 28 (5), 509 (2005).

S. CHIMICHI, M. BOCCALINI, M. M. M. HASSAN, G. VIOLA, F. DALL'ACQUA AND M. CURINI, Tetrahedron, 62, 90, (2006).

E. AKBAS, I. BERBER, A. SENER AND B. HASANOV, IL Farmaco, 60, 23 (2005).

A. TANITAME, Y. OYAMADA, K. OFUJI, H. TERAUCHI, M. KAWASAKI, M. WACHI AND J. YAMAGISHI, Bioorg. Med. Chem. Lett. 15 (19), 4299 (2005).

T. I. EL-EMARY, A. M. KAMAL EL-DEAN AND H. S. EL-KASHEF, IL Farmaco, 53, 383 (1998).

14 T. I. EL-EMARY AND E. A. BAKHITE, Pharmazie 54 (2), 106 (1999).

15 T. I. EL-EMARY, A. M. HUSSEIN AND H. S. EL-KASHEF, Pharmazie 55 (5), 356 (2000).

16 J. H. LANGE, H. H. VAN STUIVENBERG, W. VEERMAN, H. C. WALS, B. STORK, H. K. COOLEN, A. C. McCreary, T. J. Adolfs and C. G. Kruse, Bioorg. Med. Chem. Lett. 15 (21), 4794 (2005).

17 Q. ZHANG, P. MA, W. WANG, R. B. COLE AND G. WANG, Drug Metab. Dispos. 33 (4), 508 (2005).

18 F. WUST, T. KNIESS, M. KRETZSCHMAR AND R. BERGMANN, Bioorg. Med. Chem. Lett. 15 (5), 1303 (2005).

19 C. D. COX, M. J. BRESLIN, B. J. MARIANO, P. J. COLEMAN, C. A. BUSER, E. S. WALSH, K. HAMILTON, H. E. HUBER, N. E. KOHL, M. TORRENT, Y. YAN, L. C. KUO AND G. D. HARTMAN, Bioorg. Med. Chem. Lett. 15, 2041 (2005).

20 H. S. EL-KASHEF, T. I. EL-EMARY, M. GASQUET, P TIMON-DAVID, J. MALDONALDO AND P. VANELLE, Pharmazie, 55(8), 572 (2000).

21 P. RATHELOT, N. AZAS, H. EL-KASHEF, F. DELMAS, C. DI GIORGIO, P. TIMON-DAVID, J. MALDONADO AND P. VANELLE, Eur. J. Med. Chem. 37(8), 671 ( 2002).

22 S. A. LAUFER, G. K. WAGNER, D. A. KOTSCHENREUTHER AND W. ALBRECHT, J. Med. Chem., 46 (15), 3230 (2003).

23 J. C. BARROW, P. G. NANTERMET, S. R. STAUFFER, P. L. NGO, M. A. STEINBEISER, S.S. MAO, S. S. CARROLL, C. BAILEY, D. COLUSSI, M. BOSSERMAN, C. BURLEIN, J. J. COOK, G. SITKO, P. R. TILLER, C. M. MILLER-STEIN, M. ROSE, D. R. MCMASTERS, J. P. VACCA, AND H. G. SELNICK, J. Med. Chem., 46 (25), 5294 (2003).

24 K. S. ATWAL, P. WANG, W. L. ROGERS, P. SLEPH, H. MONSHIZADEGAN, F. N. FERRARA, S. TRAEGER, D. W. GREEN AND G. J. GROVER, J. Med. Chem., 47(5), 1081 (2004). 
S. SARAVANAN, P. S. SELVAN, N. GOPAL, J. K. GUPTA, B. DE. ARCH. Pharmazie 338(10), 488 (2005).

A. H. ABADI, S. M. ABOU-SERI, D. E. ABDEL-RAHMAN, CH. KLEIN, O. LOZACH AND L. MEIJER, Eur. J. Med. Chem. 41(3), 296 (2006).

A. D. BORTHWICK, D. E. DAVIES, P. F. ERTL, A. M. EXALL, T. M. HALEY, G. J. HART, D. L. JACKSON, N. R. PARRY, A. PATIKIS, N. TRIVEDI, G. G. WEINGARTEN, AND J. M. WOOLVEN, J. Med. Chem. 46(21), 4428 (2003).

B. M. Kenda, A. C. MATAGne, P. E. TAlaga, P. M. PASAU, E. DIFFERDING, B. I. LALLEMAND, A. M. FRYCIA, F. G. MOUREAU, H. V. KLITGAARD, M. R. GILLARD, B. FUKS AND P. MICHEL, J. Med. Chem., 47(3), 530 (2004).

29 X. QIAN, G.B. LIANG, D. FENG, M. FISHER, T. CRUMLEY, S. RATTRAY, P. M. DULSKI, A. GURNETT, P. S. LEAVITT, P. A. LIBERATOR, A. S. MISURA, S. SAMARAS, T. TAMAS, D. M. SCHMATZ, M. WYVRATT AND T. BIFTU, Bioorg. Med. Chem. Lett. 16 (10), 2817 (2006).

S. MAHBOOBI, E. EICHHORN, A. POPP, A. SELLMER, S. ELZ AND U. MLLMANN, Eur. J. Med. Chem. 41(2), 176 (2006).

A. MAI, S. MASSA, I. CERBARA, S. VALENTE, R. RAGNO, P. BOTTONI, R. SCATENA, P. LOIDL AND G. BROSCH, J. Med. Chem., 47(5), 1098 (2004).

M. A. A. ELNEAIRY, M. A. M. GAD-ELKAREEM AND A. M. ABDELFATTAH, Phosphorous, Sulfur and Silicon, 181(6), 1451 (2006).

M. S. A. El-GABY, A. M. SH. EL-SHARIEF, A. A. ATALLA, AND A. A. A. M. EL-ADASY, Heteroatom. Chem., 16 (3), 218 (2005).

M. S. A. EL-GABY, A. M. SH. EL-SHARIEF, A. A. ATALLA, AND A. A. A. M. EL-ADASY, Afinidad, 60 (507), 475 (2003).

M. S. A. EL-GABY, A. M. SH. EL-SHARIEF, A. A. ATALLA, AND A. A. A. M. EL-ADASY, J. Chin. Chem. Soc., 51(2), 327 (2004).

M. A. KIRA, M. O. ABDEL-REHMAAN AND K. Z. GADALLA, Tetrahedron Lett. 109 (1969).

G. E. H. Elgemeie, A. H. Elgandour, A. M. ElzANATE AND A. M. HUSSEIN, J. Chem. Research (S), 256 (1997).

38 Z. E. KANDEEL, E. A. HAFEZ, M. A. SLEIM, F. M. ABDELATIF AND M. H. ELNAGDI, Heteroatom Chem., 6(4), 305 (1995). 